\title{
Note on non-orthogonality of local curvilinear co-ordinates in a three-dimensional boundary element method
}

\author{
C. Fochesato ${ }^{1, \ddagger}$, S. T. Grilli ${ }^{2, \S}$ and P. Guyenne $e^{3, *, \dagger}$ \\ ${ }^{1}$ Centre de Mathématiques et de Leurs Applications, Ecole Normale Supérieure de Cachan, \\ 94235 Cachan cedex, France \\ ${ }^{2}$ Ocean Engineering Department, University of Rhode Island, Narragansett, RI 02882, U.S.A. \\ ${ }^{3}$ Department of Mathematics, McMaster University, Hamilton, Canada, Ont L8S $4 \mathrm{Kl}$
}

\begin{abstract}
SUMMARY
We give a more general derivation of the particle velocity and acceleration used in the numerical wave model of Grilli et al. (Int. J. Numer. Meth. Fluids 2001; 35:829-867), by expressing these quantities in a local orthogonal co-ordinate system. Computations of solitary waves propagating and breaking over a sloping bottom show that the new formulation gives better results than the former one in the latest stages of overturning. Nevertheless, both formulations are found to be as suitable for the simulation of non-overturning waves. Results on wave profiles as well as on surface and internal kinematics are presented. Copyright (c) 2004 John Wiley \& Sons, Ltd.
\end{abstract}

KEY WORDS: curvilinear co-ordinates; boundary element method; three-dimensional surface waves

\section{INTRODUCTION}

In Grilli et al. [1], a three-dimensional numerical model was proposed for the simulation of non-linear water waves over arbitrary bottom topography. This model solves the full equations for potential flow using a high-order boundary element method (BEM) and a mixed Eulerian-Lagrangian time updating. In this BEM, the boundary geometry and field variables are represented by 16-node quadrilateral elements, providing bi-cubic local interpolation of the solution in between nodes. High-order tangential derivatives, required for the time updating, are calculated in a local curvilinear co-ordinate system $(\xi, \eta)$, using 25 -node fourth-order

\footnotetext{
*Correspondence to: P. Guyenne, Department of Mathematics, McMaster University, 1280 Main Street West, Hamilton, Canada, Ont L8S 4K1.

†E-mail: guyenne@math.mcmaster.ca

${ }^{\ddagger}$ E-mail: fochesat@cmla.ens-cachan.fr

§E-mail: grilli@oce.uri.edu

Contract/grant sponsor: SHARCNET
}

Received 7 March 2004

Copyright (c) 2004 John Wiley \& Sons, Ltd. 
quadrilateral elements. This intrinsic curvilinear co-ordinate system is used to express physical quantities such as the particle velocity and acceleration at each collocation node on the boundary. The corresponding unit vectors $(\mathbf{s}, \mathbf{m}, \mathbf{n})$ are defined as derivatives of the Cartesian position vector $\mathbf{x}$ with respect to the intrinsic co-ordinates. These are introduced to map Cartesian boundary elements to a reference element of regular shape. Grilli et al. [1] assumed that the so-defined local co-ordinate system is orthogonal, which is however not true in general. The reason is that, in the mixed Eulerian-Lagrangian formulation, discretization nodes are allowed to move with the flow and boundary elements to distort as time progresses. In particular, distortions of boundary elements can be very severe when computing highly nonlinear waves like overturning waves. The main purpose of this paper is to give a more general derivation of the particle velocity and acceleration in a local orthogonal curvilinear co-ordinate system and show that more accurate results can be obtained with this new formulation.

The remainder of the paper is organized as follows. In Section 2, we review the mathematical formulation of the wave model for the convenience of the reader. We derive in addition the expressions of the internal velocity and acceleration fields, which can be evaluated from the boundary values. The new derivation of the particle velocity and acceleration on the free surface is described in Section 3. We review the numerical methods including the quadrature method for singular integrals in Section 4. Applications to solitary waves propagating and breaking over a sloping ridge, and comparisons with the former model [1] are presented in Section 5. Results on both surface and internal kinematics of overturning waves are shown.

\section{MATHEMATICAL FORMULATION}

Equations for a fully non-linear potential flow with a free surface are listed in the following. The velocity potential $\phi(\mathbf{x}, t)$ is introduced to describe an inviscid, irrotational flow in Cartesian co-ordinates $\mathbf{x}=(x, y, z)$ with $z$ the vertical upward direction $(z=0$ at the undisturbed free surface), and the fluid velocity is expressed as $\mathbf{u}=\boldsymbol{\nabla} \phi$.

The continuity equation in the fluid domain $\Omega(t)$ with boundary $\Gamma(t)$ is Laplace's equation

$$
\nabla^{2} \phi=0
$$

The corresponding three-dimensional free-space Green's function is defined as

$$
G\left(\mathbf{x}, \mathbf{x}_{l}\right)=\frac{1}{4 \pi r} \quad \text { with } \quad \frac{\partial G}{\partial n}\left(\mathbf{x}, \mathbf{x}_{l}\right)=-\frac{1}{4 \pi} \frac{\mathbf{r} \cdot \mathbf{n}}{r^{3}}
$$

where $r=|\mathbf{r}|=\left|\mathbf{x}-\mathbf{x}_{l}\right|$ is the distance from the source point $\mathbf{x}$ to the field point $\mathbf{x}_{l}$ (both on boundary $\Gamma$ ), and $\mathbf{n}$ is the outward unit vector normal to the boundary at point $\mathbf{x}$.

Green's second identity transforms Equation (1) into the boundary integral equation (BIE)

$$
\alpha\left(\mathbf{x}_{l}\right) \phi\left(\mathbf{x}_{l}\right)=\int_{\Gamma}\left[\frac{\partial \phi}{\partial n}(\mathbf{x}) G\left(\mathbf{x}, \mathbf{x}_{l}\right)-\phi(\mathbf{x}) \frac{\partial G}{\partial n}\left(\mathbf{x}, \mathbf{x}_{l}\right)\right] \mathrm{d} \Gamma
$$

where $\alpha\left(\mathbf{x}_{l}\right)=\theta_{l} /(4 \pi)$ and $\theta_{l}$ is the exterior solid angle at point $\mathbf{x}_{l}$.

The boundary is divided into various parts satisfying different boundary conditions. On the free surface, $\phi$ satisfies the non-linear kinematic and dynamic boundary conditions in the 
mixed Eulerian-Lagrangian formulation, respectively

$$
\begin{gathered}
\frac{\mathrm{D} \mathbf{R}}{\mathrm{D} t}=\mathbf{u}=\nabla \phi \\
\frac{\mathrm{D} \phi}{\mathrm{D} t}=-g z+\frac{1}{2} \nabla \phi \cdot \nabla \phi-\frac{p}{\rho}
\end{gathered}
$$

with $\mathbf{R}$ being the position vector of a fluid particle on the free surface, $g$ the acceleration due to gravity, $p$ the atmospheric pressure, $\rho$ the fluid density and $\mathrm{D} / \mathrm{D} t=\partial / \partial t+\nabla \phi \cdot \nabla$ the Lagrangian time derivative. On the bottom and other fixed parts of the boundary, a no-flow condition is prescribed as

$$
\frac{\partial \phi}{\partial n}=0
$$

Once Equation (3) is solved, the solution within the domain can be evaluated from the boundary values. Using Equation (3), the internal velocity is given by

$$
\mathbf{u}\left(\mathbf{x}_{l}\right)=\nabla \phi\left(\mathbf{x}_{l}\right)=\int_{\Gamma}\left[\frac{\partial \phi}{\partial n}(\mathbf{x}) \mathbf{Q}\left(\mathbf{x}, \mathbf{x}_{l}\right)-\phi(\mathbf{x}) \frac{\partial \mathbf{Q}}{\partial n}\left(\mathbf{x}, \mathbf{x}_{l}\right)\right] \mathrm{d} \Gamma
$$

with

$$
\mathbf{Q}\left(\mathbf{x}, \mathbf{x}_{l}\right)=\frac{1}{4 \pi r^{3}} \mathbf{r}, \quad \frac{\partial \mathbf{Q}}{\partial n}\left(\mathbf{x}, \mathbf{x}_{l}\right)=\frac{1}{4 \pi r^{3}}\left[\mathbf{n}-3(\mathbf{r} \cdot \mathbf{n}) \frac{\mathbf{r}}{r^{2}}\right]
$$

and $r$ denoting the distance from the boundary point $\mathbf{x}$ to the interior point $\mathbf{x}_{l}$. Note that the coefficient $\alpha\left(\mathbf{x}_{l}\right)$ reduces to unity for interior points.

Similarly, we can also derive the internal Lagrangian acceleration

$$
\frac{\mathrm{D} \mathbf{u}}{\mathrm{D} t}=\frac{\mathrm{D}}{\mathrm{D} t} \boldsymbol{\nabla} \phi=\frac{\partial}{\partial t} \boldsymbol{\nabla} \phi+(\boldsymbol{\nabla} \phi \cdot \nabla) \nabla \phi
$$

where the first term in the right-hand side, corresponding to the local acceleration, is given by

$$
\boldsymbol{\nabla} \frac{\partial \phi}{\partial t}\left(\mathbf{x}_{l}\right)=\int_{\Gamma}\left[\frac{\partial^{2} \phi}{\partial t \partial n}(\mathbf{x}) \mathbf{Q}\left(\mathbf{x}, \mathbf{x}_{l}\right)-\frac{\partial \phi}{\partial t}(\mathbf{x}) \frac{\partial \mathbf{Q}}{\partial n}\left(\mathbf{x}, \mathbf{x}_{l}\right)\right] \mathrm{d} \Gamma
$$

and the last term is computed using Equation (7) and differentiating $\nabla \phi$. This requires calculating the spatial derivatives for all components of $\mathbf{Q}$ and $\partial \mathbf{Q} / \partial n$. Their expressions are

$$
\begin{gathered}
\frac{\partial Q_{i}}{\partial x_{j}}= \begin{cases}\frac{3}{4 \pi r^{5}} r_{i} r_{j}, & i \neq j \\
\frac{1}{4 \pi r^{3}}\left(\frac{3}{r^{2}} r_{i}^{2}-1\right), & i=j\end{cases} \\
\frac{\partial}{\partial x_{j}}\left(\frac{\partial Q_{i}}{\partial n}\right)= \begin{cases}\frac{3}{4 \pi r^{5}}\left[r_{j} n_{i}+r_{i} n_{j}-\frac{5}{r^{2}}(\mathbf{r} \cdot \mathbf{n}) r_{i} r_{j}\right], & i \neq j \\
\frac{3}{4 \pi r^{5}}\left[\mathbf{r} \cdot \mathbf{n}+2 r_{i} n_{i}-\frac{5}{r^{2}}(\mathbf{r} \cdot \mathbf{n}) r_{i}^{2}\right], & i=j\end{cases}
\end{gathered}
$$


where $i, j$ refer to the spatial dimensions and $r_{i}$ stands for the $i$ th component of $\mathbf{r}$. For near-surface interior points, a special treatment is required to compute accurately the (quasisingular) integrals in Equations (7) and (10) (see Section 4.3). The quantities $\partial \phi / \partial t$ and $\partial^{2} \phi / \partial t \partial n$ in Equation (10) also satisfy a BIE similar to Equation (3) for $\phi$ and $\partial \phi / \partial n$. In fact, the calculation of their values on the free surface is done as part of the second-order time integration method outlined in Section 4.1.

\section{PARTICLE VELOCITY AND ACCELERATION}

In Grilli et al. [1], a local curvilinear co-ordinate system is introduced to express the geometry, field variables and their derivatives at each collocation node on the boundary. Thus, at each nodal point $\mathbf{x}$, the corresponding unit tangential vectors are defined as

$$
\mathbf{s}=\frac{1}{h_{1}} \frac{\partial \mathbf{x}}{\partial \xi} \quad \text { and } \quad \mathbf{m}=\frac{1}{h_{2}} \frac{\partial \mathbf{x}}{\partial \eta}
$$

where

$$
h_{1}=\left|\frac{\partial \mathbf{x}}{\partial \xi}\right|, \quad h_{2}=\left|\frac{\partial \mathbf{x}}{\partial \eta}\right|
$$

and $-1 \leqslant \xi, \eta \leqslant 1$ denote the intrinsic co-ordinates in the reference element. A third unit vector in the normal direction is then defined as $\mathbf{n}=\mathbf{s} \times \mathbf{m}$. Grilli et al. [1] assumed that $\mathbf{s}$ and $\mathbf{m}$ are orthogonal, and derived expressions for the particle velocity $\mathbf{u}$ and acceleration $\mathrm{D} \mathbf{u} / \mathrm{D} t$ in the co-ordinate system $(\mathbf{s}, \mathbf{m}, \mathbf{n})$. The orthogonality of $\mathbf{s}$ and $\mathbf{m}$ however does not hold when the boundary elements are distorted, as in regions of large surface deformations. Hereafter, we give the derivation of tangential derivatives in the general case where $\mathbf{s}$ and $\mathbf{m}$ are not orthogonal. It should be noted that this aspect of the discretization only concerns the time updating of the boundary conditions on the free surface. It does not affect the solution of the BEM.

Using $\mathbf{s}$ and $\mathbf{m}$ in Equation (13), one can define a new unit tangential vector as (see Figure 1)

$$
\mathbf{m}^{\prime}=\frac{1}{\sqrt{1-\kappa^{2}}} \mathbf{m}-\frac{\kappa}{\sqrt{1-\kappa^{2}}} \mathbf{S}
$$

so that $\mathbf{s}$ and $\mathbf{m}^{\prime}$ are orthogonal (i.e. $\mathbf{s} \cdot \mathbf{m}^{\prime}=0$ ) with $\kappa=\mathbf{s} \cdot \mathbf{m}$. This implies that $-1<\kappa<1$. The unit normal vector now takes the form

$$
\mathbf{n}=\mathbf{s} \times \mathbf{m}^{\prime}=\frac{1}{\sqrt{1-\kappa^{2}}} \mathbf{S} \times \mathbf{m}
$$

One can see that $\mathbf{m}^{\prime}=\mathbf{m}$ and $\mathbf{s} \cdot \mathbf{m}=0$ only when $\kappa=0$. For clarity, let us introduce the following notations:

$$
()_{s} \equiv \frac{\partial}{\partial s}=\frac{1}{h_{1}} \frac{\partial}{\partial \xi}, \quad()_{m} \equiv \frac{\partial}{\partial m}=\frac{1}{h_{2}} \frac{\partial}{\partial \eta}, \quad()_{n} \equiv \frac{\partial}{\partial n}
$$




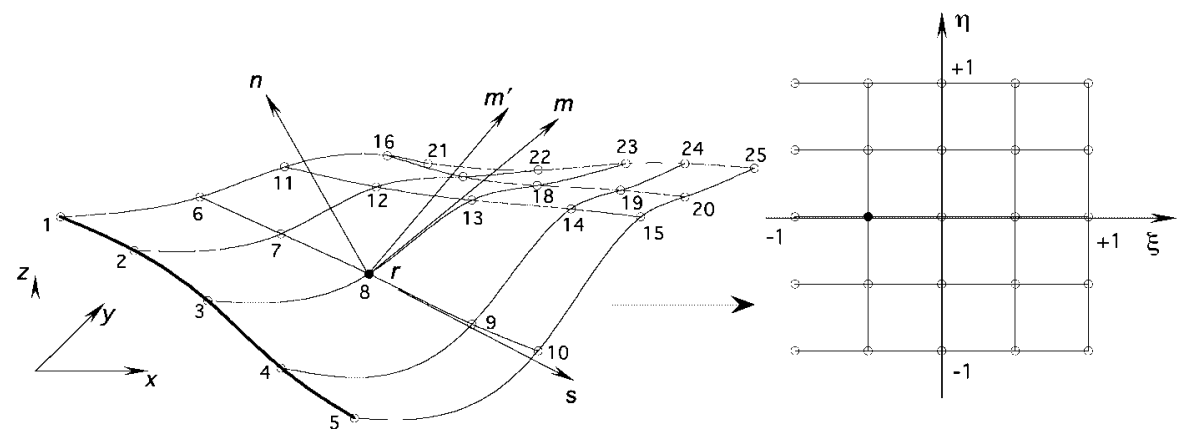

Figure 1. Sketch of local interpolation by fourth-order two-dimensional sliding polynomials of $(\xi, \eta)$, for calculating tangential derivatives in orthogonal axis $\left(\mathbf{s}, \mathbf{m}^{\prime}, \mathbf{n}\right)$ at point $\mathbf{r}$ on the boundary.

and

$$
()_{s s} \equiv \frac{1}{h_{1}^{2}} \frac{\partial^{2}}{\partial \xi^{2}}, \quad()_{s m} \equiv \frac{1}{h_{1} h_{2}} \frac{\partial^{2}}{\partial \xi \partial \eta}, \quad()_{m m} \equiv \frac{1}{h_{2}^{2}} \frac{\partial^{2}}{\partial \eta^{2}}
$$

In the new orthonormal co-ordinate system $\left(\mathbf{s}, \mathbf{m}^{\prime}, \mathbf{n}\right)$, the particle velocity on the boundary is expressed as

$$
\mathbf{u}=\nabla \phi=\phi_{s} \mathbf{s}+\phi_{m^{\prime}} \mathbf{m}^{\prime}+\phi_{n} \mathbf{n}
$$

Returning to the co-ordinate system $(\mathbf{s}, \mathbf{m}, \mathbf{n})$, Equation (19) is given by

$$
\nabla \phi=\frac{1}{1-\kappa^{2}}\left(\phi_{s}-\kappa \phi_{m}\right) \mathbf{s}+\frac{1}{1-\kappa^{2}}\left(\phi_{m}-\kappa \phi_{s}\right) \mathbf{m}+\phi_{n} \mathbf{n}
$$

after using Equation (15) and the fact that

$$
\phi_{m^{\prime}}=\frac{1}{\sqrt{1-\kappa^{2}}} \phi_{m}-\frac{\kappa}{\sqrt{1-\kappa^{2}}} \phi_{s}
$$

Laplace's equation $\nabla^{2} \phi=0$ can be similarly expressed, which leads to

$$
\begin{aligned}
\phi_{n n}= & -\frac{1}{1-\kappa^{2}}\left\{\phi_{s s}+\phi_{m m}-2 \kappa \phi_{s m}\right\} \\
& +\frac{\phi_{s}}{\left(1-\kappa^{2}\right)^{2}}\left\{\mathbf{x}_{s s} \cdot \mathbf{s}+\mathbf{x}_{m m} \cdot \mathbf{s}-\kappa \mathbf{x}_{s s} \cdot \mathbf{m}-\kappa \mathbf{x}_{m m} \cdot \mathbf{m}-2 \kappa \mathbf{x}_{s m} \cdot \mathbf{s}+2 \kappa^{2} \mathbf{x}_{s m} \cdot \mathbf{m}\right\} \\
& +\frac{\phi_{m}}{\left(1-\kappa^{2}\right)^{2}}\left\{\mathbf{x}_{s s} \cdot \mathbf{m}+\mathbf{x}_{m m} \cdot \mathbf{m}-\kappa \mathbf{x}_{s s} \cdot \mathbf{s}-\kappa \mathbf{x}_{m m} \cdot \mathbf{s}-2 \kappa \mathbf{x}_{s m} \cdot \mathbf{m}+2 \kappa^{2} \mathbf{x}_{s m} \cdot \mathbf{s}\right\} \\
& +\frac{\phi_{n}}{1-\kappa^{2}}\left\{\mathbf{x}_{s s} \cdot \mathbf{n}+\mathbf{x}_{m m} \cdot \mathbf{n}-2 \kappa \mathbf{x}_{s m} \cdot \mathbf{n}\right\}
\end{aligned}
$$


Substituting Equation (22) into the expression for the particle acceleration on the boundary yields

$$
\begin{aligned}
& \frac{\mathrm{Du}}{\mathrm{D} t}=\left(\frac{\partial}{\partial t}+\nabla \phi \cdot \nabla\right) \nabla \phi \\
& =\mathbf{s}\left[\frac{1}{1-\kappa^{2}}\left\{\phi_{t s}-\kappa \phi_{t m}+\phi_{n} \phi_{n s}-\kappa \phi_{n} \phi_{n m}\right\}\right. \\
& +\frac{1}{\left(1-\kappa^{2}\right)^{2}}\left\{\phi_{s} \phi_{s s}-2 \kappa \phi_{s} \phi_{s m}+\kappa^{2} \phi_{s} \phi_{m m}\right. \\
& \left.-\kappa \phi_{m} \phi_{m m}+\left(1+\kappa^{2}\right) \phi_{m} \phi_{s m}-\kappa \phi_{m} \phi_{s s}\right\} \\
& +\frac{\phi_{s}^{2}}{\left(1-\kappa^{2}\right)^{3}}\left\{-\mathbf{x}_{s s} \cdot \mathbf{s}+\kappa \mathbf{x}_{s s} \cdot \mathbf{m}+2 \kappa \mathbf{x}_{s m} \cdot \mathbf{s}\right. \\
& \left.-2 \kappa^{2} \mathbf{x}_{s m} \cdot \mathbf{m}-\kappa^{2} \mathbf{x}_{m m} \cdot \mathbf{S}+\kappa^{3} \mathbf{x}_{m m} \cdot \mathbf{m}\right\} \\
& +\frac{\phi_{s} \phi_{m}}{\left(1-\kappa^{2}\right)^{3}}\left\{2 \kappa \mathbf{x}_{S S} \cdot \mathbf{s}-\left(1+\kappa^{2}\right) \mathbf{x}_{s S} \cdot \mathbf{m}-\left(1+3 \kappa^{2}\right) \mathbf{x}_{s m} \cdot \mathbf{s}\right. \\
& \left.+\kappa\left(3+\kappa^{2}\right) \mathbf{x}_{s m} \cdot \mathbf{m}+\kappa\left(1+\kappa^{2}\right) \mathbf{x}_{m m} \cdot \mathbf{s}-2 \kappa^{2} \mathbf{x}_{m m} \cdot \mathbf{m}\right\} \\
& +\frac{\phi_{m}^{2}}{\left(1-\kappa^{2}\right)^{3}}\left\{-\kappa^{2} \mathbf{x}_{s S} \cdot \mathbf{s}+\kappa \mathbf{x}_{s S} \cdot \mathbf{m}+\kappa\left(1+\kappa^{2}\right) \mathbf{x}_{s m} \cdot \mathbf{s}\right. \\
& \left.-\left(1+\kappa^{2}\right) \mathbf{x}_{s m} \cdot \mathbf{m}-\kappa^{2} \mathbf{x}_{m m} \cdot \mathbf{s}+\kappa \mathbf{x}_{m m} \cdot \mathbf{m}\right\} \\
& +\frac{\phi_{s} \phi_{n}}{\left(1-\kappa^{2}\right)^{2}}\left\{-\kappa^{2} \mathbf{x}_{s s} \cdot \mathbf{n}+2 \kappa \mathbf{x}_{s m} \cdot \mathbf{n}-\kappa^{2} \mathbf{x}_{m m} \cdot \mathbf{n}\right\} \\
& \left.+\frac{\phi_{m} \phi_{n}}{\left(1-\kappa^{2}\right)^{2}}\left\{\kappa \mathbf{x}_{s s} \cdot \mathbf{n}-\left(1+\kappa^{2}\right) \mathbf{x}_{s m} \cdot \mathbf{n}+\kappa^{3} \mathbf{x}_{m m} \cdot \mathbf{n}\right\}\right] \\
& +\mathbf{m}\left[\frac{1}{1-\kappa^{2}}\left\{-\kappa \phi_{t s}+\phi_{t m}-\kappa \phi_{n} \phi_{n s}+\phi_{n} \phi_{n m}\right\}\right. \\
& +\frac{1}{\left(1-\kappa^{2}\right)^{2}}\left\{-\kappa \phi_{s} \phi_{s s}+\left(1+\kappa^{2}\right) \phi_{s} \phi_{s m}-\kappa \phi_{s} \phi_{m m}\right. \\
& \left.+\phi_{m} \phi_{m m}-2 \kappa \phi_{m} \phi_{s m}+\kappa^{2} \phi_{m} \phi_{s s}\right\} \\
& +\frac{\phi_{s}^{2}}{\left(1-\kappa^{2}\right)^{3}}\left\{\kappa \mathbf{x}_{s s} \cdot \mathbf{s}-\kappa^{2} \mathbf{x}_{s s} \cdot \mathbf{m}-\left(1+\kappa^{2}\right) \mathbf{x}_{s m} \cdot \mathbf{s}\right. \\
& \left.+\kappa\left(1+\kappa^{2}\right) \mathbf{x}_{s m} \cdot \mathbf{m}+\kappa \mathbf{x}_{m m} \cdot \mathbf{s}-\kappa^{2} \mathbf{x}_{m m} \cdot \mathbf{m}\right\} \\
& +\frac{\phi_{s} \phi_{m}}{\left(1-\kappa^{2}\right)^{3}}\left\{-2 \kappa^{2} \mathbf{x}_{s s} \cdot \mathbf{s}+\kappa\left(1+\kappa^{2}\right) \mathbf{x}_{s s} \cdot \mathbf{m}+\kappa\left(3+\kappa^{2}\right) \mathbf{x}_{s m} \cdot \mathbf{s}\right.
\end{aligned}
$$




$$
\begin{aligned}
& \left.-\left(1+3 \kappa^{2}\right) \mathbf{x}_{s m} \cdot \mathbf{m}-\left(1+\kappa^{2}\right) \mathbf{x}_{m m} \cdot \mathbf{s}+2 \kappa \mathbf{x}_{m m} \cdot \mathbf{m}\right\} \\
& +\frac{\phi_{m}^{2}}{\left(1-\kappa^{2}\right)^{3}}\left\{\kappa^{3} \mathbf{x}_{S S} \cdot \mathbf{s}-\kappa^{2} \mathbf{x}_{S S} \cdot \mathbf{m}-2 \kappa^{2} \mathbf{x}_{s m} \cdot \mathbf{s}\right. \\
& \left.+2 \kappa \mathbf{x}_{s m} \cdot \mathbf{m}+\kappa \mathbf{x}_{m m} \cdot \mathbf{s}-\mathbf{x}_{m m} \cdot \mathbf{m}\right\} \\
& +\frac{\phi_{s} \phi_{n}}{\left(1-\kappa^{2}\right)^{2}}\left\{\kappa^{3} \mathbf{x}_{s s} \cdot \mathbf{n}-\left(1+\kappa^{2}\right) \mathbf{x}_{s m} \cdot \mathbf{n}+\kappa \mathbf{x}_{m m} \cdot \mathbf{n}\right\} \\
& \left.+\frac{\phi_{m} \phi_{n}}{\left(1-\kappa^{2}\right)^{2}}\left\{-\kappa^{2} \mathbf{x}_{s s} \cdot \mathbf{n}+2 \kappa \mathbf{x}_{s m} \cdot \mathbf{n}-\kappa^{2} \mathbf{x}_{m m} \cdot \mathbf{n}\right\}\right] \\
& +\mathbf{n}\left[\phi_{t n}+\frac{1}{1-\kappa^{2}}\left\{\phi_{s} \phi_{n s}-\kappa \phi_{s} \phi_{n m}-\kappa \phi_{m} \phi_{n s}\right.\right. \\
& \left.+\phi_{m} \phi_{n m}-\phi_{n} \phi_{s s}+2 \kappa \phi_{n} \phi_{s m}-\phi_{n} \phi_{m m}\right\} \\
& +\frac{\phi_{s}^{2}}{\left(1-\kappa^{2}\right)^{2}}\left\{\mathbf{x}_{s s} \cdot \mathbf{n}-2 \kappa \mathbf{x}_{s m} \cdot \mathbf{n}+\kappa^{2} \mathbf{x}_{m m} \cdot \mathbf{n}\right\} \\
& +\frac{2 \phi_{s} \phi_{m}}{\left(1-\kappa^{2}\right)^{2}}\left\{-\kappa \mathbf{x}_{s s} \cdot \mathbf{n}+\left(1+\kappa^{2}\right) \mathbf{x}_{s m} \cdot \mathbf{n}-\kappa \mathbf{x}_{m m} \cdot \mathbf{n}\right\} \\
& +\frac{\phi_{m}^{2}}{\left(1-\kappa^{2}\right)^{2}}\left\{\kappa^{2} \mathbf{x}_{S S} \cdot \mathbf{n}-2 \kappa \mathbf{x}_{s m} \cdot \mathbf{n}+\mathbf{x}_{m m} \cdot \mathbf{n}\right\} \\
& +\frac{\phi_{S} \phi_{n}}{\left(1-\kappa^{2}\right)^{2}}\left\{\mathbf{x}_{S S} \cdot \mathbf{s}-\kappa \mathbf{x}_{S S} \cdot \mathbf{m}-2 \kappa \mathbf{x}_{s m} \cdot \mathbf{s}\right. \\
& \left.+2 \kappa^{2} \mathbf{x}_{s m} \cdot \mathbf{m}+\mathbf{x}_{m m} \cdot \mathbf{s}-\kappa \mathbf{x}_{m m} \cdot \mathbf{m}\right\} \\
& +\frac{\phi_{m} \phi_{n}}{\left(1-\kappa^{2}\right)^{2}}\left\{-\kappa \mathbf{x}_{s S} \cdot \mathbf{s}+\mathbf{x}_{s S} \cdot \mathbf{m}+2 \kappa^{2} \mathbf{x}_{s m} \cdot \mathbf{s}\right. \\
& \left.-2 \kappa \mathbf{x}_{s m} \cdot \mathbf{m}-\kappa \mathbf{x}_{m m} \cdot \mathbf{s}+\mathbf{x}_{m m} \cdot \mathbf{m}\right\} \\
& \left.+\frac{\phi_{n}^{2}}{1-\kappa^{2}}\left\{\mathbf{x}_{s s} \cdot \mathbf{n}-2 \kappa \mathbf{x}_{s m} \cdot \mathbf{n}+\mathbf{x}_{m m} \cdot \mathbf{n}\right\}\right]
\end{aligned}
$$

When $\kappa=0$, formulas (20), (22) and (23) reduce to

$$
\begin{gathered}
\mathbf{u}=\phi_{s} \mathbf{s}+\phi_{m} \mathbf{m}+\phi_{n} \mathbf{n} \\
\phi_{n n}=-\phi_{s s}-\phi_{m m}+\phi_{s}\left(\mathbf{x}_{s s} \cdot \mathbf{s}-\mathbf{x}_{s m} \cdot \mathbf{m}\right)+\phi_{m}\left(\mathbf{x}_{m m} \cdot \mathbf{m}-\mathbf{x}_{s m} \cdot \mathbf{s}\right) \\
+\phi_{n}\left(\mathbf{x}_{s s} \cdot \mathbf{n}+\mathbf{x}_{m m} \cdot \mathbf{n}\right)
\end{gathered}
$$


and

$$
\begin{aligned}
\frac{\mathrm{Du}}{\mathrm{D} t}= & \mathbf{s}\left[\phi_{t s}+\phi_{s} \phi_{s s}+\phi_{m} \phi_{s m}+\phi_{n} \phi_{n s}-\phi_{s}^{2} \mathbf{x}_{s s} \cdot \mathbf{s}\right. \\
& \left.+\phi_{m}^{2} \mathbf{x}_{m m} \cdot \mathbf{s}-\phi_{m} \phi_{n} \mathbf{x}_{s m} \cdot \mathbf{n}\right] \\
& +\mathbf{m}\left[\phi_{t m}+\phi_{s} \phi_{s m}+\phi_{m} \phi_{m m}+\phi_{n} \phi_{n m}+\phi_{s}^{2} \mathbf{x}_{s s} \cdot \mathbf{m}\right. \\
& \left.-\phi_{m}^{2} \mathbf{x}_{m m} \cdot \mathbf{m}-\phi_{s} \phi_{n} \mathbf{x}_{s m} \cdot \mathbf{n}\right] \\
& +\mathbf{n}\left[\phi_{t n}+\phi_{s} \phi_{n s}+\phi_{m} \phi_{n m}-\phi_{n}\left(\phi_{s s}+\phi_{m m}\right)+\phi_{s}^{2} \mathbf{x}_{s s} \cdot \mathbf{n}\right. \\
& +\phi_{m}^{2} \mathbf{x}_{m m} \cdot \mathbf{n}+2 \phi_{s} \phi_{m} \mathbf{x}_{s m} \cdot \mathbf{n}+\phi_{n}^{2}\left(\mathbf{x}_{s s} \cdot \mathbf{n}+\mathbf{x}_{m m} \cdot \mathbf{n}\right) \\
& \left.+\phi_{s} \phi_{n}\left(\mathbf{x}_{s s} \cdot \mathbf{s}-\mathbf{x}_{s m} \cdot \mathbf{m}\right)+\phi_{m} \phi_{n}\left(\mathbf{x}_{m m} \cdot \mathbf{m}-\mathbf{x}_{s m} \cdot \mathbf{s}\right)\right]
\end{aligned}
$$

which are the expressions given in Reference [1] (Equations (60)-(62)). Note that, when $\mathbf{s} \cdot \mathbf{m}=0$, one has the identities $\mathbf{x}_{s s} \cdot \mathbf{m}=-\mathbf{x}_{s m} \cdot \mathbf{s}$ and $\mathbf{x}_{s m} \cdot \mathbf{m}=-\mathbf{x}_{m m} \cdot \mathbf{s}$.

\section{NUMERICAL METHODS}

We only summarize here the methods for temporal and spatial discretizations. Since the only difference between the former and new models lies in the calculation of tangential derivatives on the boundary, the reader is referred to Reference [1] for more details on the numerical methods and applications to solitary wave shoaling and breaking.

\subsection{Time integration}

A second-order explicit scheme based on Taylor series expansions is used to update the position $\mathbf{R}$ and velocity potential $\phi$ on the free surface, as

$$
\begin{aligned}
& \mathbf{R}(t+\Delta t)=\mathbf{R}+\Delta t \frac{\mathrm{D} \mathbf{R}}{\mathrm{D} t}+\frac{\Delta t^{2}}{2} \frac{\mathrm{D}^{2} \mathbf{R}}{\mathrm{D} t^{2}}+O\left(\Delta t^{3}\right) \\
& \phi(t+\Delta t)=\phi+\Delta t \frac{\mathrm{D} \phi}{\mathrm{D} t}+\frac{\Delta t^{2}}{2} \frac{\mathrm{D}^{2} \phi}{\mathrm{D} t^{2}}+O\left(\Delta t^{3}\right)
\end{aligned}
$$

where $\Delta t$ is the varying time step and all terms in the right-hand sides are evaluated at time $t$. First-order coefficients in these Taylor series are given by Equations (4) and (5), which requires calculating $\phi, \partial \phi / \partial n$ at time $t$ on the free surface. Second-order coefficients are obtained from the Lagrangian time derivative of Equations (4) and (5), which also requires calculating $\partial \phi / \partial t, \partial^{2} \phi / \partial t \partial n$ at time $t$. Since the BIEs to be solved in both cases correspond to the same boundary geometry, one needs to discretize and assemble the resulting linear system only once. 
Global accuracy of the numerical scheme can be assessed at any time by checking the conservation of volume

$$
V=\int_{\Gamma} z n_{z} \mathrm{~d} \Gamma
$$

and total energy

$$
E=\frac{1}{2} \rho \int_{\Gamma}\left(\phi \frac{\partial \phi}{\partial n}+g z^{2} n_{z}\right) \mathrm{d} \Gamma
$$

where the first and second terms represent the kinetic and potential contributions of the flow respectively, and $n_{z}$ is the vertical component of the unit normal vector.

\subsection{Boundary discretization}

A high-order three-dimensional BEM is used to solve numerically the BIEs for $\phi$ and $\partial \phi / \partial t$. The boundary is discretized into collocation nodes, defining two-dimensional elements for local interpolations of the solution in between these nodes. Thus, within each element, the boundary geometry and field variables are interpolated using polynomial shape functions. The boundary elements are $4 \times 4$-node quadrilaterals associated with bi-cubic shape functions, ensuring local $C^{2}$ continuity at their edges.

The discretized boundary integrals are evaluated for each collocation node by numerical integration. When the collocation node does not belong to the integrated element, a standard Gauss-Legendre quadrature is applied. When it belongs to the element, the distance $r$ in the Green's function and in its normal gradient vanishes at one of the nodes of the element. For such singular situations, a method of singularity extraction is used based on polar co-ordinate transformations. Tangential derivatives in Equations (27) and (28) are calculated by defining fourth-order $5 \times 5$-node elements.

As the linear algebraic system resulting from the discretization of the BIE (3) for $\phi$ (and $\partial \phi / \partial t)$ is in general dense and non-symmetric, a generalized minimal residual (GMRES) algorithm with preconditioning is used to solve it. We point out that we used the same discretization and integration methods to solve the BIEs and compute the internal kinematics given by the integral expressions (7) and (10).

\subsection{Quadrature method for quasi-singular integrals}

Integrals in Equation (3) may have a highly varying kernel when the distance $r$ becomes small (albeit non-zero) in the neighbourhood of a collocation point. These are then referred to as quasi-singular integrals and a standard Gauss quadrature, with a fixed number of integration points, may fail to accurately calculate such integrals. Grilli and Subramanya [2] showed, for two-dimensional problems, that the loss of accuracy of Gauss integrations may be several orders of magnitude, when the distance to the collocation point becomes very small. This loss of accuracy should be more severe in three dimensions due to the fact that $G \sim 1 / r$ (compared with $G \sim \ln r$ in two dimensions).

Situations of quasi-singular integrals generally occur near intersections of boundary parts (e.g. between the free surface and lateral boundaries) or in regions of the free surface like breaker jets. For such cases, Grilli et al. [1] developed an adaptive method based on a binary subdivision of the integrated element. Integrals over the so-defined sub-elements are then 
calculated using a standard Gauss quadrature (with 10 integration points) after some additional co-ordinate transformations. To identify possible quasi-singular integrals in the discretized domain and choose the adequate number of subdivisions, both distance and intercept angle thresholds are checked for each reference point.

Quasi-singular integrals may also occur, even more severely, in the calculation of the internal velocity and Lagrangian acceleration for interior points close to the boundary (e.g. just beneath the water surface or inside the breaker jet). As shown in Equations (8) and (12), the kernels grow very fast like $1 / r^{3}$ for $\mathbf{u}$ and $1 / r^{4}$ for $\partial \mathbf{u} / \partial t$ as $r$ tends to zero. Here, for the accurate treatment of such integrals, we applied an adaptive scheme similar to that in Reference [1]. This method, however, can be computationally costly, especially when large numbers of subdivisions are needed for interior points very close to the boundary. In our applications, we found that two binary subdivisions (i.e. 16 sub-elements) suffice in most cases.

\section{NUMERICAL RESULTS AND COMPARISONS}

To test the performance of the model with the new expressions for $\mathbf{u}$ and $\mathrm{Du} / \mathrm{D} t$, we carry out computations of solitary waves propagating and breaking over a sloping ridge. Computed results and their accuracy are assessed by comparison with those obtained from the former three-dimensional model in Reference [1] and the two-dimensional model of Grilli et al. [3]. Results on both surface and internal kinematics of overturning waves are shown.

\subsection{Comparison with the former three-dimensional model}

The bottom topography is specified as follows: a constant depth $h_{0}$ in the first part of the domain and then a sloping ridge starting at $x^{\prime}=5.225$, with a $1: 15$ slope in the middle and a transverse modulation of the form $\operatorname{sech}^{2}\left(k y^{\prime}\right)(k=0.5)$. The computational domain is $4 h_{0}$ wide and $19 h_{0}$ long, with vertical walls specified at its extremities and sides. Primes hereafter indicate non-dimensional variables based on long wave theory, i.e. lengths are divided by the

reference depth $h_{0}$ and times by $\sqrt{h_{0} / g}$, where $g$ is the acceleration due to gravity. The initial condition is an exact 'Tanaka' solitary wave of height $H_{0}^{\prime}=0.6$.

The initial discretization for the bottom and free surface consists of $40 \times 10$ quadrilateral elements in the $x$ - and $y$-directions respectively (with regular grid sizes $\Delta x_{0}^{\prime}=0.47, \Delta y_{0}^{\prime}=0.40$ ). The initial time step is set to $\Delta t_{0}^{\prime}=0.18$. Computations are first performed in this discretization up to $t^{\prime}=5.7$ and regridding to a finer resolution is then applied in the portion $8.075 \leqslant x^{\prime} \leqslant 19$ $\left(\Delta x^{\prime}=0.27, \Delta y^{\prime}=0.40\right)$. Figure 2 shows the wave profiles at $t^{\prime}=8.995$ and $t^{\prime}=9.142$ using the old (in Reference [1]) and new formulas, respectively. We stress that the same experimental parameters are used in both cases. One can see that both solutions reach an advanced stage of wave overturning. However, computations with the new formulas break down at a later time than those with the old formulas. The break down of computations is due to situations of quasi-singular integrals, associated with the phenomenon of node convergence in the breaking jet.

Figure 3 shows the comparison of relative errors on energy and volume conservation for the two models. In both cases, the errors exhibit similar temporal behaviours. Although there is a gradual growth in time, they remain small up to the last computed time (less than $1 \%$ ). 


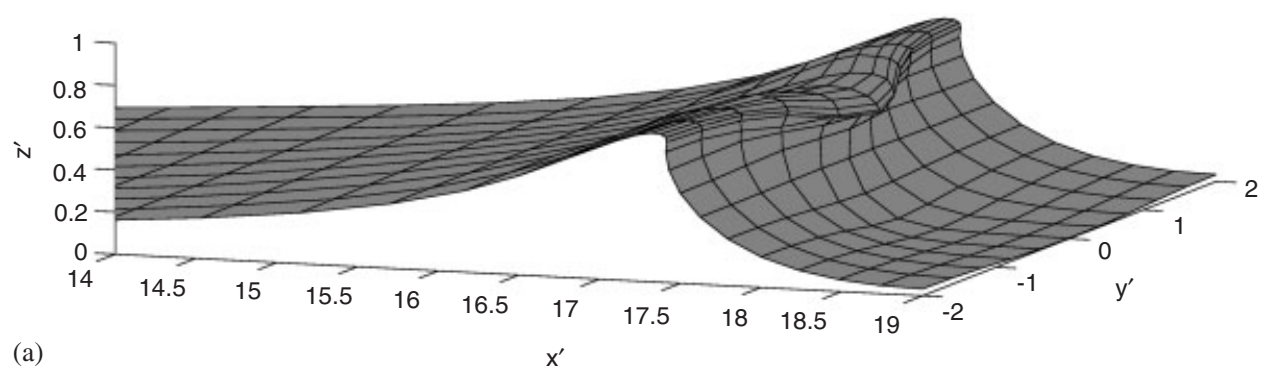

(a)

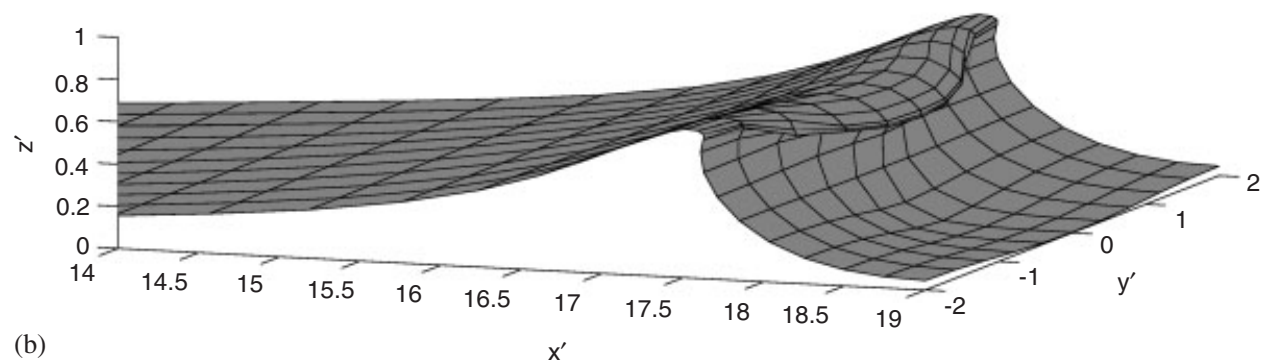

Figure 2. Wave profiles in the last computed stage at (a) $t^{\prime}=8.995$ (old formulas) and (b) $t^{\prime}=9.142$ (new formulas). The discretization is $40 \times 10$ elements in the $x$ - and $y$-directions, respectively.
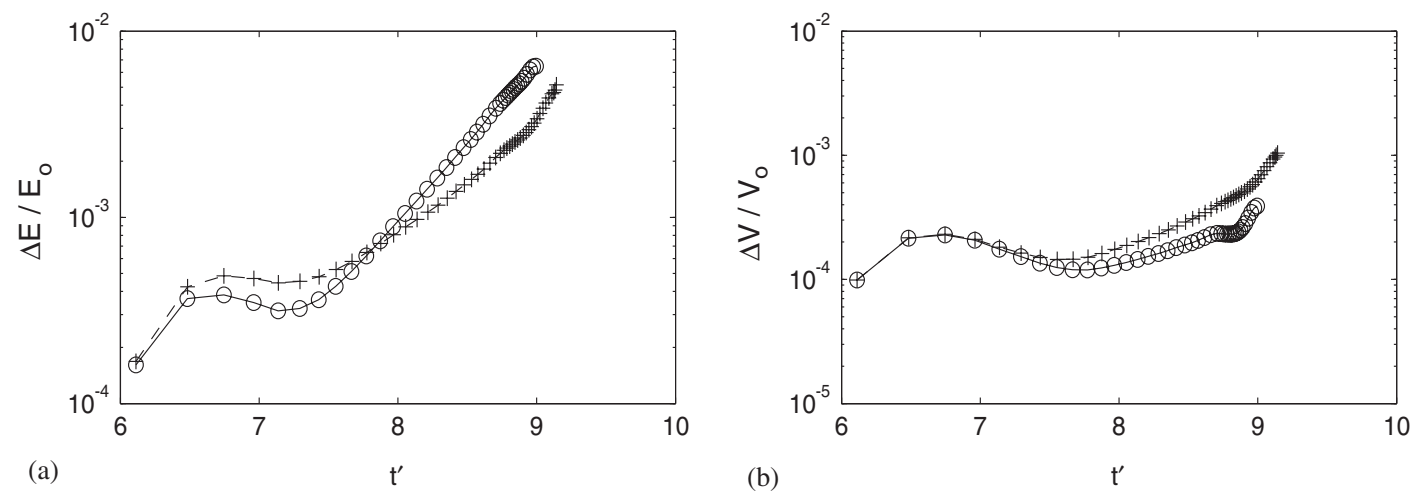

Figure 3. Time evolution of relative errors on (a) energy and (b) volume conservation, using the old (solid line-circles) and new (dashed line-crosses) formulas, after regridding at $t^{\prime}=5.7$.

The reference energy $E_{0}$ and volume $V_{0}$ correspond to the energy and volume levels at the regridding time $t^{\prime}=5.7$.

In Figure 4, we present a direct comparison in the middle cross-section $y^{\prime}=0$ at three different stages of evolution, for which the two solutions exhibit similar free surface profiles. It can be seen that the two wave profiles match almost perfectly up to the breaking point (Figure 4(b)). The corresponding times are also nearly the same. However, as wave break- 

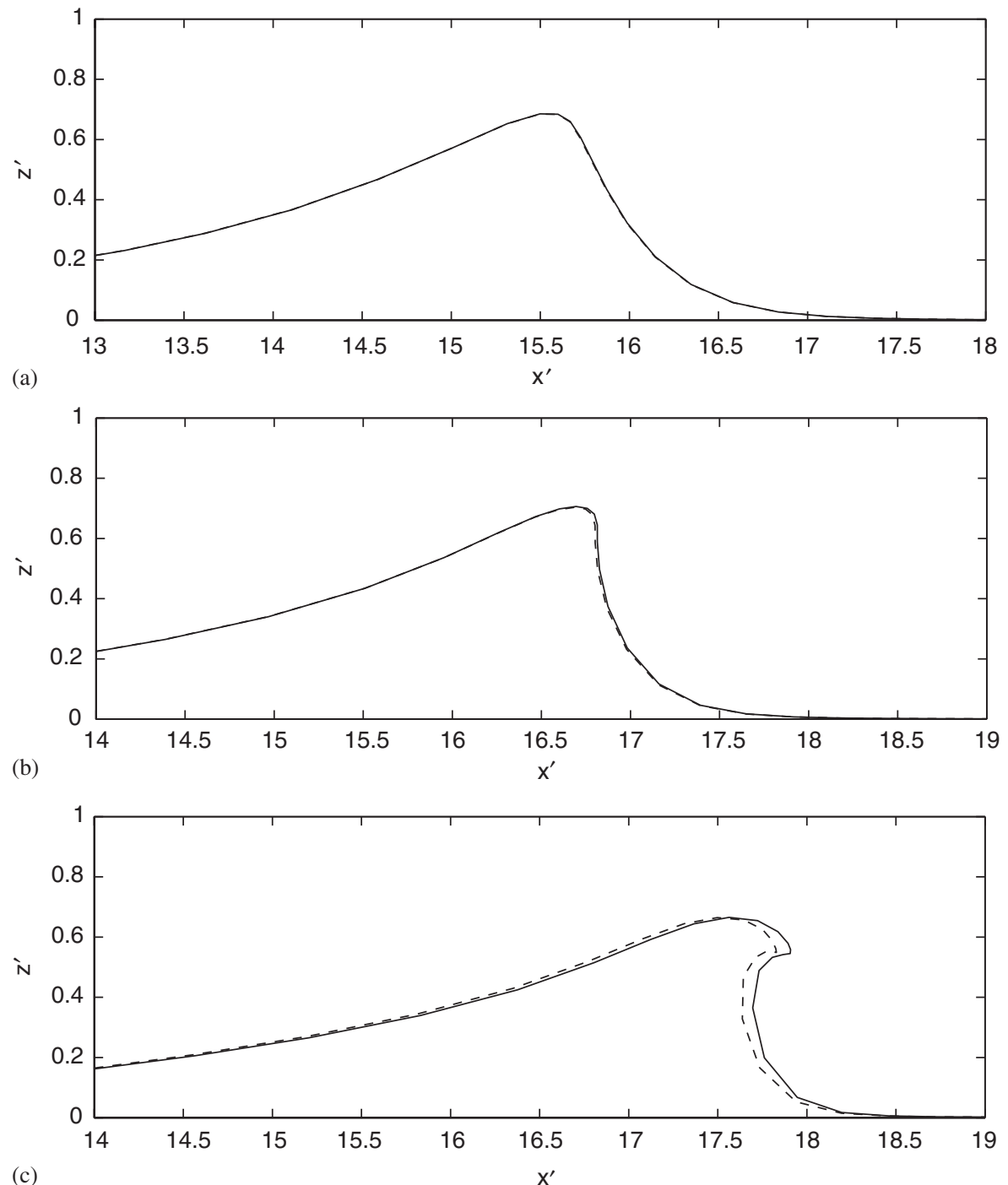

Figure 4. Comparison of wave profiles in the cross-section $y^{\prime}=0$ using the old, new formulas (dashed, solid lines) at (a) $t^{\prime}=7.551,7.554$ and (b) $t^{\prime}=8.349,8.356$ (breaking point) and (c) $t^{\prime}=8.995,9.045$, respectively.

ing develops, differences in time evolution and wave profiles become more significant. The breaking jet seems to develop slightly faster when using the old formulas.

It is important to notice that both models give very similar results up to the breaking point. As long as the free surface does not undergo significant deformations, the mesh remains nearly regular and the vectors $\mathbf{s}$ and $\mathbf{m}$ remain nearly orthogonal at all collocation nodes. Indeed, as shown in Figure 5 (using the new formulas), the maximum magnitude of $\mathbf{s} \cdot \mathbf{m}$ for nodes on the free surface gradually increases with time, to reach only $\sim 0.4$ at the breaking point $\left(t^{\prime}=8.356\right)$. It then increases faster (with oscillations) as the wave overturns. 


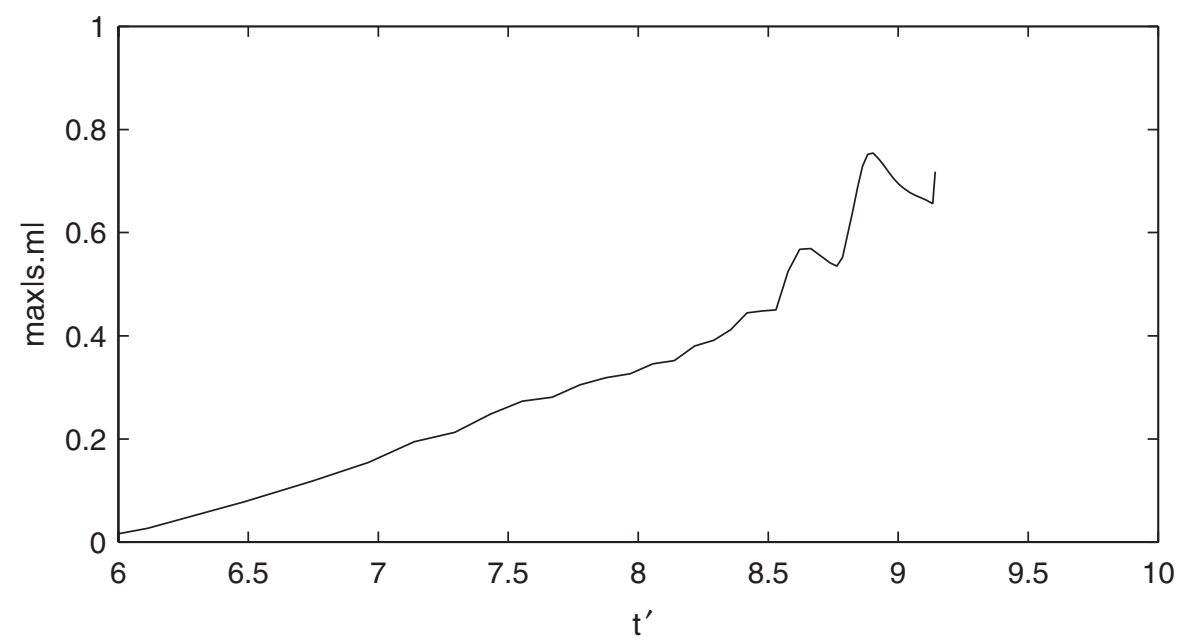

Figure 5. Time evolution for the maximum of $|\mathbf{s} \cdot \mathbf{m}|$ on the free surface, after regridding at $t^{\prime}=5.7$. The new formulas for the particle velocity and acceleration are used here.

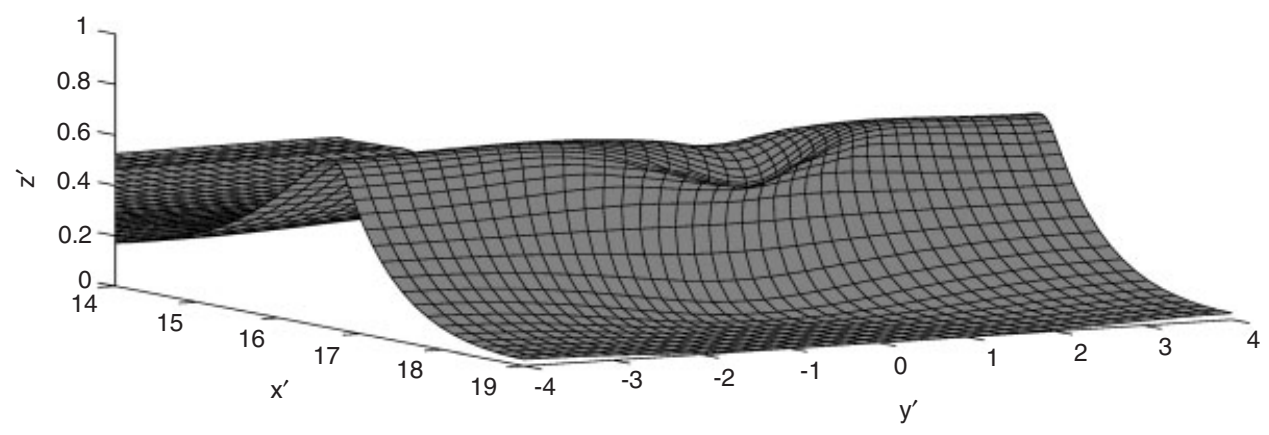

Figure 6. Wave profile in the latest computed stage at $t^{\prime}=8.600$, using the old formulas. The discretization is $60 \times 40$ elements in the $x$ - and $y$-directions, respectively.

Therefore, we can infer that both formulations are as equally suitable up to the breaking point and that formulas (24)-(26) provide very good approximations for smooth nonoverturning waves.

In order to better appreciate the differences between both models, we now perform computations for a larger domain and a finer resolution. The length of the domain is $19 h_{0}$ but its width is increased to $8 h_{0}(k=0.25)$. The initial wave is the same 'Tanaka' solitary wave of height $H_{0}^{\prime}=0.6$ as before. The initial discretization is $50 \times 20$ elements $\left(\Delta x_{0}^{\prime}=0.38, \Delta y_{0}^{\prime}=0.40\right)$, and it is increased to $60 \times 40$ elements $\left(\Delta x^{\prime}=0.18, \Delta y^{\prime}=0.20\right)$ when regridding on $8.075 \leqslant x^{\prime} \leqslant 19$ at $t^{\prime}=4$.9. Figures 6 and 7 display the wave profiles at $t^{\prime}=8.600$ and 8.904,9.142, using the old and new formulas, respectively. High-resolution computations with the new formulas 

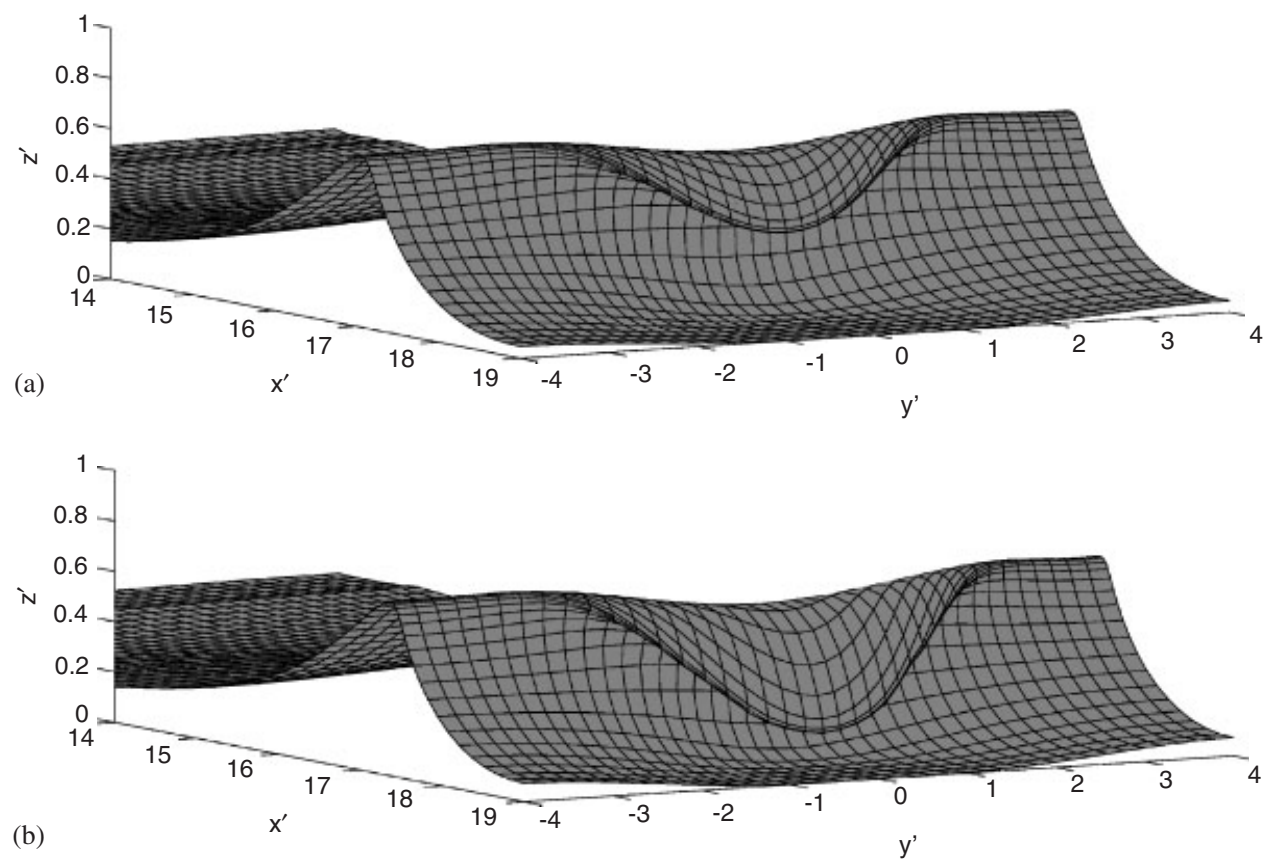

Figure 7. Wave profiles in the latest computed stages at (a) $t^{\prime}=8.904$ and (b) $t^{\prime}=9.142$, using the new formulas. The discretization is $60 \times 40$ elements in the $x$ - and $y$-directions, respectively.

clearly reach a more advanced stage of wave overturning than those with the old formulas. In the former case, the smoother transverse variation for the tip of the jet better reflects the smooth transverse variation of the bottom geometry. The breaking process can be computed up to near the impact of the jet onto the forward face of the wave (Figure 7(b)).

In Figure 8, the comparison of errors reveals that volume is well conserved throughout the calculations by both models $(\sim 0.01 \%)$. There is however a large improvement of up to one order of magnitude regarding the conservation of energy. Indeed, the corresponding errors are within $0.1 \%$ with the new formulation, while they almost reach $1 \%$ with the old one. The faster accumulation of errors in the latter case explains why computations break down earlier as mentioned above.

We would like to point out that, in all our computations, no (explicit) smoothing/filtering was used to stabilize the solution. In particular, we never observed the so-called sawtooth instabilities on the free surface as reported in e.g. Longuet-Higgins and Cokelet [4].

\subsection{Comparison with the two-dimensional model}

We also found it useful to compare the new model with the two-dimensional version of Grilli et al. [3,5]. This two-dimensional model has been extensively tested and validated against both theoretical and experimental results. In particular, in the case of shoaling and breaking of solitary waves on plane slopes, it has been shown that the computations of surface 

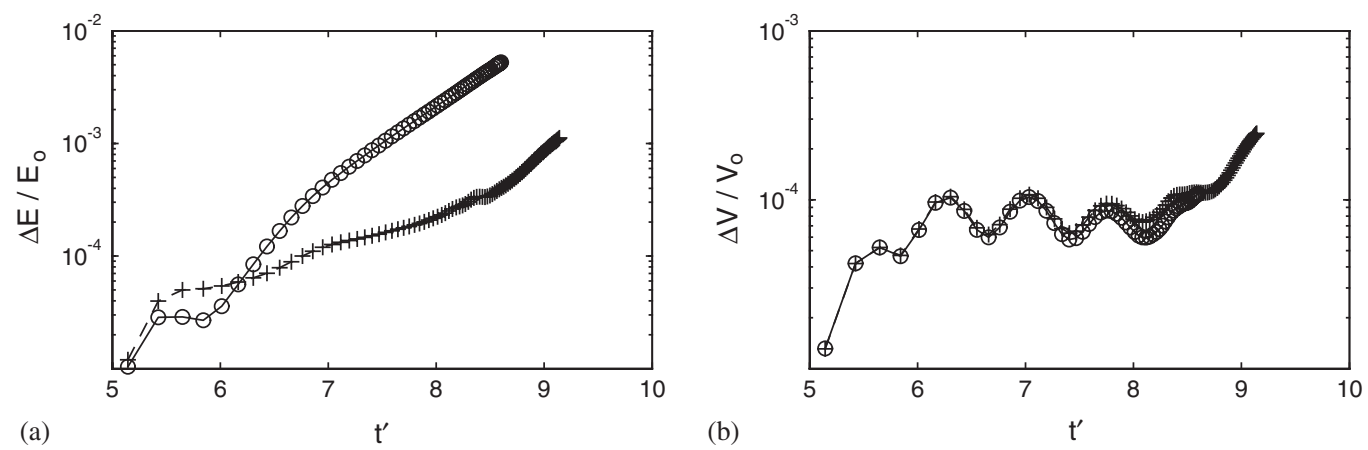

Figure 8. Time evolution of relative errors on (a) energy and (b) volume conservation, using the old (solid line-circles) and new (dashed line-crosses) formulas, after regridding at $t^{\prime}=4.9$.

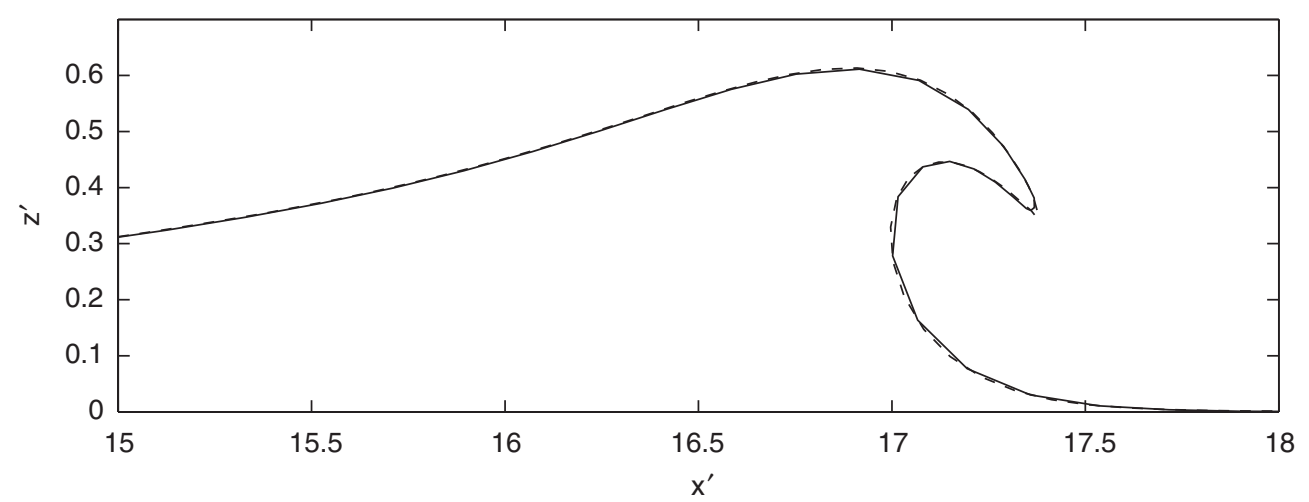

Figure 9. Comparison between three-dimensional (solid line) and two-dimensional (dashed line) results in the vertical cross-section $y^{\prime}=0$ at $t^{\prime}=8.560$. The discretization in the $x$-direction is $\Delta x^{\prime}=0.10,0.18$ for the two-, three-dimensional waves, respectively.

elevations agreed to within $2 \%$ with laboratory measurements up to the breaking point (see References $[3,6])$.

Here, we specify a narrow three-dimensional domain of width $2 h_{0}$ with no transverse variation in the $y$-direction. Both two- and three-dimensional simulations are run for a $1: 15$ bottom slope and an initial solitary wave of height $H_{0}^{\prime}=0.6$, and the corresponding discretizations in the $x$-direction are $\Delta x^{\prime}=0.10,0.18$, respectively. Figure 9 shows the comparison between two- and three-dimensional results in the middle cross-section $y^{\prime}=0$ at $t^{\prime}=8.560$. One can see that the two wave profiles match almost perfectly, even in the region of the breaking jet. A similar comparison was already made in Reference [1] but could not be performed for such an advanced stage of wave overturning. This good agreement supports the ability (and superiority) of the new model to describe large surface deformations, even with moderate resolutions. 


\subsection{Kinematics of three-dimensional overturning waves}

We present here some results on the surface and internal kinematics associated with overturning waves. The particle velocity $\mathbf{u}=\nabla \phi=\left(u_{x}, u_{y}, u_{z}\right)$ and acceleration $\mathbf{a}=\mathrm{D} \mathbf{u} / \mathrm{D} t=\left(a_{x}, a_{y}, a_{z}\right)$ on the free surface are computed using Equations (20) and (23). For interior points, we use the integral expressions (7) and (9).

Figure 10 shows the surface velocity and acceleration fields $\left(u_{x}, u_{z}\right)$ and $\left(a_{x}, a_{z}\right)$ in the middle cross-section $y^{\prime}=0$. The solution at the latest time $t^{\prime}=9.142$ is considered here (Figure 7(b)). For comparison, the linear phase speed in shallow water $c=\sqrt{g h_{0}}$ and the gravity acceleration $g$ denote the unit speed and acceleration in our non-dimensionalization. The maximum velocity occurs near the tip of the jet and is found to be $\left|\mathbf{u}^{\prime}\right|=1.997$. The maximum acceleration $\left(\left|\mathbf{a}^{\prime}\right|=4.867\right)$ occurs on the front face of the wave. The near free-fall motion of the plunging jet is clearly indicated by fluid particles with accelerations close to $g$ and directed downward. Note that some acceleration vectors at the tip of the jet with unacceptable magnitude and direction are omitted in Figure 10(b). This is because the calculation of particle accelerations is very sensitive to local variations of the boundary geometry (as shown in Equation (23)), and therefore inaccuracies are expected to be more significant in the overturning region.

Overall, we find a good agreement with existing results of two-dimensional numerical simulations of overturning waves (e.g. on uniform depth by New et al. [7] and in deep water by Dommermuth et al. [8]). These authors observed maximum velocities as high as almost $2 c$ and maximum accelerations up to $5-6 \mathrm{~g}$ on the free surface.

Figure 11 displays the internal velocity and acceleration fields $\left(u_{x}, u_{z}\right)$ and $\left(a_{x}, a_{z}\right)$ in the middle cross-section $y^{\prime}=0$ at $t^{\prime}=9.142$. The interior points are chosen equally spaced by $\Delta x_{i}^{\prime}=0.10$ and $\Delta z_{i}^{\prime}=0.05$ along the $x$ - and $z$-axis respectively. The maximum velocity (resp. acceleration) that we computed is $\left|\mathbf{u}^{\prime}\right|=1.974$ (resp. $\left|\mathbf{a}^{\prime}\right|=2.204$ ). The quasi-uniformity of the velocity distribution along the depth is clearly shown in Figure 11(a), which is expected for a very long wave such as a solitary wave. In contrast, the acceleration field has appreciable values only in the region adjacent to the wave front face (Figure 11(b)).
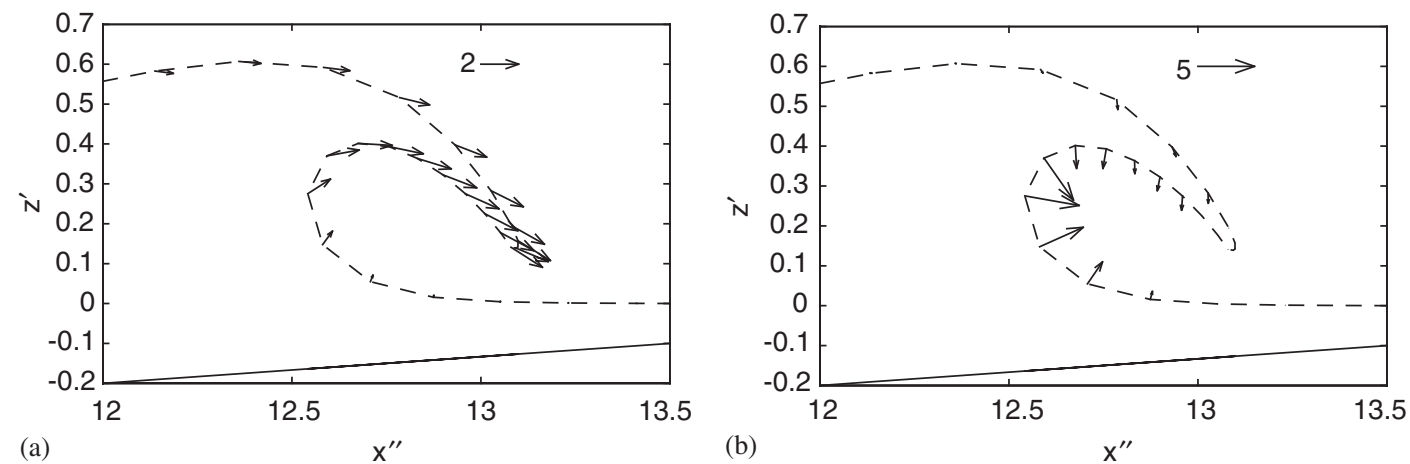

Figure 10. Vertical cross-sections $\left(y^{\prime}=0\right)$ of the surface velocity (a) and acceleration (b) fields at $t^{\prime}=9.142$. The $x$-axis is shifted to $x^{\prime \prime}=x^{\prime}-d_{0}^{\prime}\left(d_{0}^{\prime}=5.225\right)$. The straight line represents the bottom profile. In the $(z, x)$-plane, the maximum velocity (resp. acceleration) is $\left|\mathbf{u}^{\prime}\right|=1.997$ (resp. $\left|\mathbf{a}^{\prime}\right|=4.867$ ). 


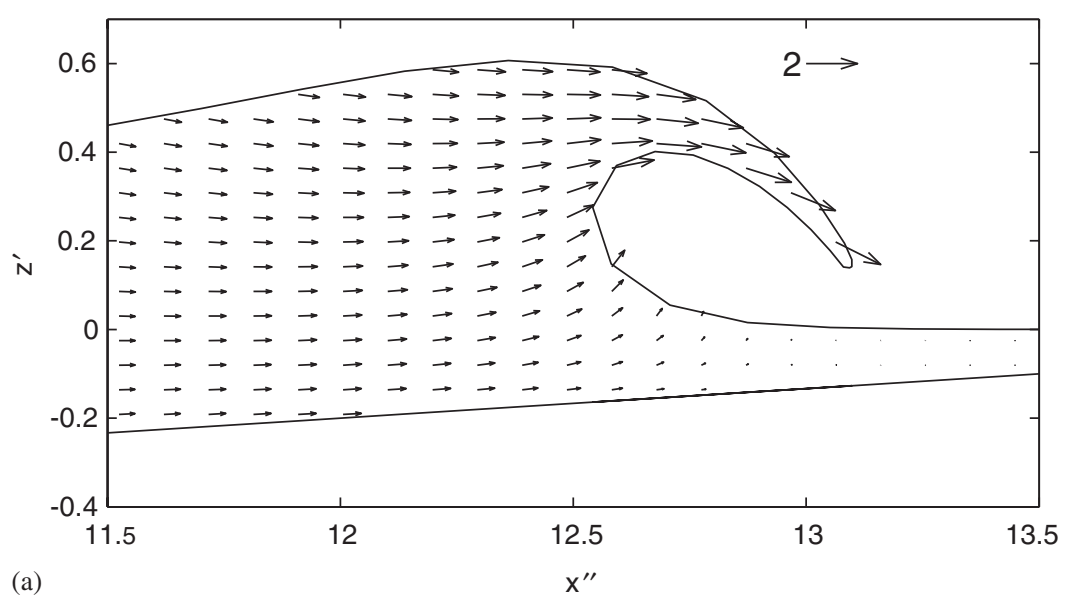

(a)

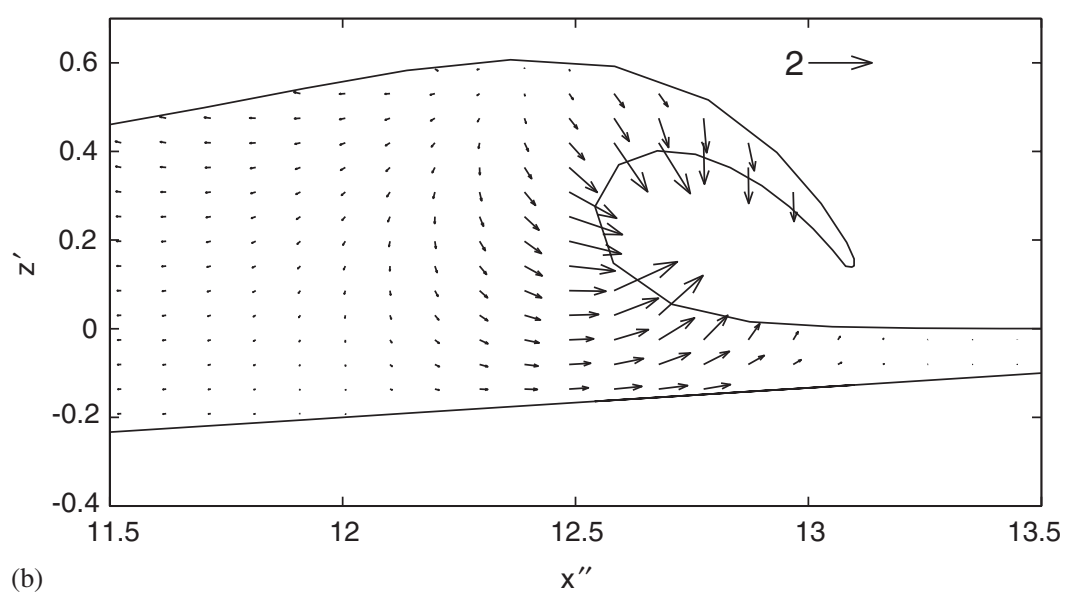

Figure 11. Vertical cross-sections $\left(y^{\prime}=0\right)$ of the internal velocity (a) and acceleration (b) fields at $t^{\prime}=9.142$. The $x$-axis is shifted to $x^{\prime \prime}=x^{\prime}-d_{0}^{\prime}\left(d_{0}^{\prime}=5.225\right)$. The straight line represents the bottom profile. In the $(z, x)$-plane, the maximum velocity (resp. acceleration) is $\left|\mathbf{u}^{\prime}\right|=1.974\left(\right.$ resp. $\left.\left|\mathbf{a}^{\prime}\right|=2.204\right)$.

Transverse variations of the wave kinematics are illustrated in Figure 12, where we plot the internal velocity and acceleration fields $\left(u_{x}, u_{y}\right)$ and $\left(a_{x}, a_{y}\right)$ at $z^{\prime}=-0.2\left(\Delta x_{i}^{\prime}=0.35\right.$ and $\left.\Delta y_{i}^{\prime}=0.38\right)$. The maximum velocity (resp. acceleration) that we computed is $\left|\mathbf{u}^{\prime}\right|=0.694$ (resp. $\left|\mathbf{a}^{\prime}\right|=0.589$ ). As indicated, the propagation of the solitary wave is associated with a forward displacement of water beneath the surface. There are some small transverse variations of both velocity and acceleration fields due to focusing of the flow by the ridge.

Our results on internal kinematics of overturning waves are consistent with those obtained by Biausser et al. [9], who used a volume of fluid/Navier-Stokes solver to simulate threedimensional wave breaking on slopes. 

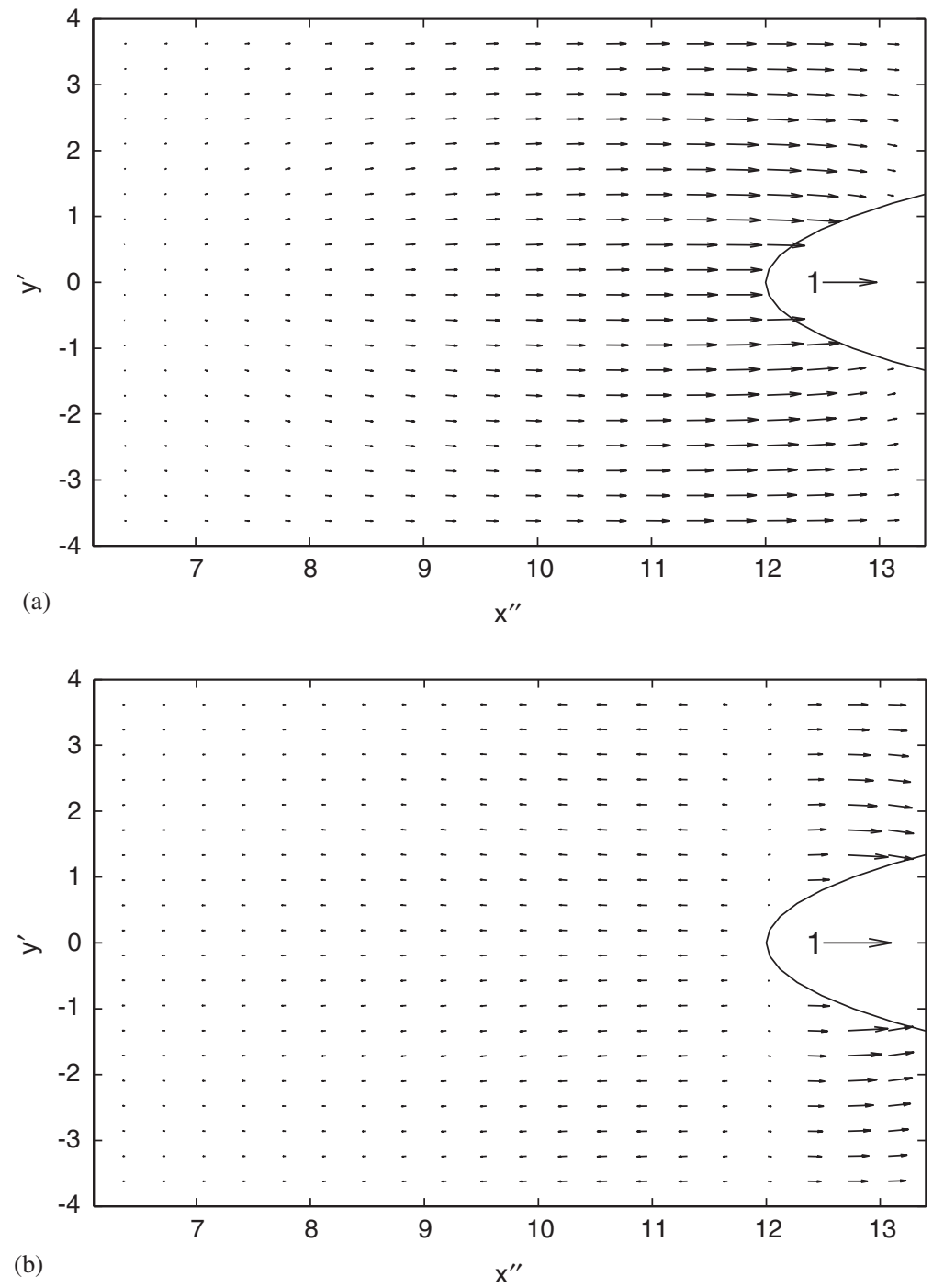

Figure 12. Horizontal cross-sections $\left(z^{\prime}=-0.2\right)$ of the internal velocity (a) and acceleration (b) fields at $t^{\prime}=9.142$. The $x$-axis is shifted to $x^{\prime \prime}=x^{\prime}-d_{0}^{\prime}\left(d_{0}^{\prime}=5.225\right)$. The solid curve at the right extremity represents the cross-section of the sloping ridge. In the $(x, y)$-plane, the maximum velocity (resp. acceleration) is $\left|\mathbf{u}^{\prime}\right|=0.694$ (resp. $\left.\left|\mathbf{a}^{\prime}\right|=0.589\right)$.

\section{CONCLUSIONS}

A more general derivation of tangential derivatives used in the numerical wave model of Grilli et al. [1] has been given by considering a local orthogonal co-ordinate system. We have derived new expressions for the particle velocity and acceleration on the free surface, which are required for the time updating. As a test, computations of solitary waves propagating 
and breaking over a sloping bottom have been carried out. Our results show a significant improvement on the former model [1] for the simulation of overturning waves. Computations with the new model are more accurate in terms of conservation of energy, and more stable in the sense that, for a given grid resolution, they reach a more advanced stage of wave breaking. Nevertheless, we find that the two formulations give very similar and accurate results in the case of non-overturning waves.

The kinematics of overturning waves have also been examined, both on the free surface and within the flow. Our results for maximum velocity $(\sim 2 c)$ and acceleration $(\sim 5 g)$ computed on the free surface are consistent with the two-dimensional results reported by New et al. [7] and Dommermuth et al. [8]. In fact, there are strong similarities in both wave profiles and kinematics between plunging breakers in the present case and those computed in the two works cited previously.

The present model has been shown to be suitable for the numerical simulation of highly nonlinear three-dimensional water waves, like overturning waves. A few typical results of physical interest, that can be calculated with our model, have been analysed. More in-depth numerical computations have been made, to achieve a better understanding of these phenomena. Since such computations constitute a substantial contribution in their own right, they will be reported in a separate paper.

Finally, it should be mentioned that distortions of boundary elements can be greatly reduced by increasing the grid resolution, which suggests that the same results could be obtained using the old formulas with a finer mesh. This would require, however, to specify a prohibitively large number of nodes on the free surface, especially when computing strongly non-linear waves. Our calculations already span several hundreds of time steps and the CPU time is typically $O(10)$ minutes per time step on a single processor of a Compaq Alpha GS160 computer. As far as the resolutions we used here are concerned, we observe no substantial improvement when refining the spatial discretization in the former model (compare Figures 2(a) and 6). This has also been shown in Reference [1]. Hence, we conclude that the new formulation should be used when modelling highly non-linear and overturning waves.

\section{ACKNOWLEDGEMENTS}

The authors thank Yiruo Lin (Shanghai Jiao Tong University) for helpful discussions. P.G. gratefully acknowledges financial support and disposal of computing resources from SHARCNET.

\section{REFERENCES}

1. Grilli ST, Guyenne P, Dias F. A fully non-linear model for three-dimensional overturning waves over an arbitrary bottom. International Journal for Numerical Methods in Fluids 2001; 35:829-867.

2. Grilli ST, Subramanya R. Quasi-singular integrals in the modelling of nonlinear water waves in shallow water. Engineering Analysis with Boundary Elements 1994; 13:181-191.

3. Grilli ST, Svendsen IA, Subramanya R. Breaking criterion and characteristics for solitary waves on slopes. Journal of Waterway, Port, Coastal and Ocean Engineering 1997; 123:102-112.

4. Longuet-Higgins MS, Cokelet ED. The deformation of steep surface waves on water. I. A numerical method of computation. Proceedings of the Royal Society of London A 1976; 350:1-26.

5. Grilli ST, Subramanya R. Numerical modeling of wave breaking induced by fixed or moving boundaries. Computational Mechanics 1996; 17:374-391.

6. Grilli ST, Subramanya R, Svendsen IA, Veeramony J. Shoaling of solitary waves on plane beaches. Journal of Waterway, Port, Coastal and Ocean Engineering 1994; 120:609-628.

7. New AL, McIver P, Peregrine DH. Computations of overturning waves. Journal of Fluid Mechanics 1985; 150:233-251. 
8. Dommermuth DG, Yue DKP, Lin WM, Rapp RJ, Chan ES, Melville WK. Deep-water plunging breakers: a comparison between potential theory and experiments. Journal of Fluid Mechanics 1988; 189:423-442.

9. Biausser B, Grilli ST, Fraunié P. Numerical analysis of the internal kinematics and dynamics of three-dimensional breaking waves on slopes. Proceedings of the 13th International Offshore and Polar Engineering Conference, Honolulu, USA, May 2003; 340-346. 